ARTICULO DE REVISION Rev Chil Salud Pública 2013;

Vol 17 (1): 54-58

\section{Prevención del consumo de tabaco en adolescentes en base a conductas motivacionales y el análisis de campañas en Chile}

\section{RESUMEN}

Los fumadores tienen una probabilidad dos veces mayor de morir en forma prematura en comparación con los individuos que no fuman. Un número creciente de enfermedades forman la lista actual

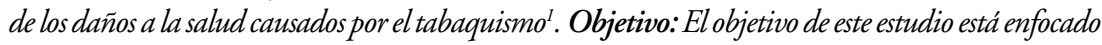
hacia la prevención del consumo de tabaco realizando un análisis de cuáles son las conductas que llevan al individuo especificamente en adolescentes, a dejar este hábito. Metodología: Fue en base a revisiones bibliográficas de artículos publicados en revistas cientificas y de salud, desde el año 2000, teniendo como motor de búsqueda el consumo de tabaco y las motivaciones para dejarlo. Discusión: La misión de los profesionales de la salud no solo está enfocada a curar enfermedades sino que también a la prevención de éstas, y el consumo de tabaco es un hábito que aumenta los riesgos de

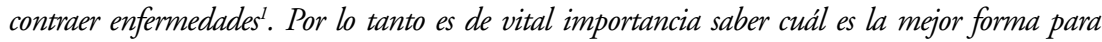
abordar la prevención contra el tabaquismo a edades tempranas, y llegar a un cambio de conducta saludable y radical. Conclusión: Las campañas antitabaco enfocadas a las motivaciones son positivas para la supresión del hábito de fumar, y pueden ser utilizadas como una herramienta para llevar a cabo la prevención de conductas no deseables, que llevan a enfermedades a futuro.

Palabras claves: Tabaquismo, prevención, motivaciones, cambios de conductas.

\section{Prevention of tobacco use in adolescents through MOTIVATIONAL BEHAVIOR CHANGE AND ANALYSIS OF CAMPAIGNS IN CHILE}

\begin{abstract}
Smokers are twice as likely to die early compared to individuals that don't smoke. The list of diseases that are known to be caused by tobacco continues to grow. Objective: Analyze which behaviors are related to smoking cessation among adolescents, in order to prevent continued tobacco consumption. Methodology: A literature review was carried out of articles published in scientific journals since 2000 on motivations to quit smoking. Discussion: The mission of health professionals is not just to heal disease, but also prevent it. Tobacco consumption significantly increases the risk of disease. Therefore, it is of vital importance to find the best way to prevent tobacco use at early ages, and promote healthy habits. Conclusion: Motivational anti-tobacco campaigns can reduce tobacco use among adolescents, and should be used as a tool for preventing unhealthy habits that will lead to future illness.
\end{abstract}

Key words: Tobacco, prevention, motivation, behavior change. 


\section{INTRODUCCIÓN}

Se conoce que el impacto del tabaco en el organismo es totalmente nocivo, existen innumerables consecuencias perjudiciales para la salud, y por supuesto enfermedades asociadas al consumo de tabaco como la Enfermedad Pulmonar Obstructiva Crónica (EPOC), cáncer al pulmón, problemas de gestación, entre otras ${ }^{2}$. Pero los profesionales de la salud tienen claro cómo abordar este problema, la labor de estos profesionales no sólo se debe orientar a la curación y rehabilitación de enfermedades sino que también deben ser capaces de desarrollar estrategias de prevención, por lo tanto es muy importante la actitud preventiva del personal de salud (médicos, enfermeras, kinesiólogos) para dejar el consumo de tabaco, y así llegar a un rol más activo y directo.

Ahora bien el hábito de fumar se consolida en la adolescencia, en donde la edad de inicio varía según las encuestas, aunque la edad media es de 14,51-3. Después se produce la consolidación del hábito y la instauración de la adicción ${ }^{2}$. Este período de la adolescencia está marcado por la necesidad de cambios en los hábitos y en el comportamiento del joven, para posteriormente consolidar su identidad. Ello motiva que se trate de una etapa de transición, en la que influidos por factores medioambientales, personales y del entorno del adolescente, se introducen hábitos tóxicos como el consumo de tabaco ${ }^{2}$.

Por lo tanto es de vital importancia erradicar la conducta tabáquica a edades tempranas y que los profesionales de la salud conozcan a qué apuntar sus estrategias preventivas, es por ello que este estudio ha enfocado su objetivo en la prevención del consumo de tabaco realizando un análisis de cuáles son las conductas que llevan al individuo específicamente en adolescentes, ha dejar este hábito. Teniendo como base el impacto de las campañas antitabaco de Chile de acuerdo a las cifras entregadas en el "octavo estudio nacional de drogas en población escolar en Chile 2009.”3.

Según Floyd, Prentice-Dunn y Rogers (2000), la teoría de la motivación para la protección ha surgido como un modelo sólido de promoción de la salud, especialmente para la prevención de enfermedades mortales importantes, uno de los componentes incorporados a la teoría de la moti- vación para la protección fueron los efectos de la recompensa intrínseca y extrínseca ${ }^{4}$. Estas teorías motivacionales son las que Chile ha ocupado en sus campañas antitabaco para llegar a la población.

Una de estas campañas se realizó hace años atrás y abordando como eje principal los daños a la salud producidos por el consumo de tabaco, colocando la fotografía de un hombre que fue laringectomizado, e imágenes de impotencia en las cajetillas de cigarro, de gran cobertura televisiva, la cual de acuerdo a la encuesta nacional de salud no fue un impacto que perduró en el tiempo ${ }^{5}$.

Una segunda campaña que hoy en día se lleva a cabo en Chile apunta a los jóvenes de $8^{\circ}$ básico a $2^{\circ}$ medio, en donde la metodología ya no es la televisión, ni las imágenes de las cajetillas, ha variado a un método más individualista, en cuanto al sector que pretende abarcar $^{6}$.

Además, se han implementado una serie de políticas ${ }^{6}$ que promuevan los ambientes libres de tabaco las que apuntan a la prohibición de fumar en restaurantes, bares y en el lugar de trabajo.

\section{METODOLOGÍA}

La metodología a utilizar en este documento fue en base a revisiones bibliográficas de artículos publicados en revistas científicas y de salud, desde el año 2001, teniendo como motor de búsqueda el consumo de tabaco y las motivaciones para dejarlo.

\section{MARCO TEÓRICO}

La teoría de las motivaciones, parece ser la mejor para la explicación del cambio de conducta, ya que ésta se refiere al proceso de las tendencias conductuales del individuo, tanto de aproximación como de evitación hacia un objeto, para sentir satisfacción. Se conocen 3 grandes campos de análisis: las motivaciones de origen fisiológico (sistema nervioso y endocrino), las motivaciones del campo de las emociones, y las motivaciones intrínsecas y extrínsecas (necesidades humanas y conductismo $)^{7}$. 
Las motivaciones intrínsecas y extrínsecas, son las de mayor recurrencia a la hora de modificar una conducta. Las primeras se refieren a las fuerzas que comandan las conductas de las personas desde su control interno de la salud, autoeficacia, autodeterminación, persistencia y las segundas se basan en el condicionamiento clásico y operante respondiendo a fuerzas externas (ambiente) como la recompensa, el castigo y el incentivo $^{7}$.

En Chile el Ministerio de Salud ha tomado como base las motivaciones intrínsecas y extrínsecas para desarrollar una serie de normas que apun$\tan$ a la mejora de conductas mal adaptativas relacionadas con la salud, específicamente antitabáquicas. Con el propósito de detener el avance del consumo de tabaco y proteger a la población de la exposición al humo de tabaco de segunda mano, ha considerado oportuno y necesario promover el desarrollo de ambientes libres de humo de tabaco en el país. Durante la primera mitad del decenio se focalizó el quehacer en el desarrollo e implementación de políticas de ambientes libres de humo de tabaco y promoción de los estilos de vida sin tabaco a nivel escolar; y en el desarrollo de un marco jurídico que facilitara el abordaje amplio e integral del control del tabaco incluyendo cambios estructurales en el país ${ }^{6}$.

El hecho de observar que el $80 \%$ de los fumadores inicia esta conducta antes de los 18 años de edad, ha movilizado alianzas de organizaciones de salud para buscar formas que permitan detener esta pandemia. Todas estas estrategias tienen como meta reducir la prevalencia de fumadores a través de dos caminos: aumentar el número de personas que se mantienen sin fumar, y aumentar el número de personas que dejan de fumar (WHO 2000) ${ }^{7}$.

En el caso de Chile ha realizado en los últimos años dos campañas que tienen como objetivo la prevención del consumo de tabaco. La primera campaña apunta al ámbito intrínseco y es realizada desde el año 2006 la cual implementaba fotografías en las cajetillas de cigarro, pasando por un hombre sin laringe, mala dentadura, impotencia, entre otras, lo cual no alcanzó el nivel de impacto esperado por las autoridades, pero según los datos entregados por el "octavo estudio nacional de drogas en población escolar en Chile 2009 ", el consumo de tabaco en cuanto a la prevalencia del último mes, el uso de cigarrillos baja de $42 \%$ a $33 \%$, en escolares de $8^{\circ}$ básico a $4^{\circ}$ medio ${ }^{3}$.
Una segunda campaña organizada por el Ministerio de Salud en conjunto con el Ministerio de Educación y el apoyo de CONAF, se encuentra en desarrollo para erradicar la conducta de fumar en adolescentes, se lleva a cabo desde el $2007^{6}$ y que apunta directamente al ámbito extrínseco, se trata de motivar a los jóvenes de $8^{\circ}$ a $2^{\circ}$ medio, mediante un concurso para dejar de fumar, la duración es de dos meses en donde los alumnos participantes deben realizar una serie de actividades, además de someterse a un test de nicotina en orina. Cabe destacar que los premios son en dinero para los cursos ganadores.

Floyd, Prentice-Dunn y Rogers a partir de su metaanálisis de una investigación basada en sesenta y cinco estudios y en más de veinte aspectos de salud ${ }^{14}$ argumentan que las intenciones y la conducta adaptativa son favorecidas por los siguientes factores: Aumento de la gravedad percibida de una amenaza para la salud; vulnerabilidad del individuo a esa amenaza; efectividad percibida de adoptar una acción protectora y autoeficacia ${ }^{14}$.

En cuanto a la decisión de continuar fumando o dejar de hacerlo, visto desde esta teoría de la motivación para la protección, considera dos procesos cognitivos que median esta decisión:

1. Reconocimiento de la amenaza; fumar tiene recompensas intrínsecas (p. ej.: Sabor en la boca, efecto de la nicotina) y recompensas extrínsecas (p. ej.: Los amigos piensan que es divertido y está de onda $)^{14}$.

2. Reconocimiento de la manera de sobreponerse; se toman en cuenta los factores de eficacia de la respuesta (si el tratamiento de reemplazo de la nicotina podría ser útil) y la autoeficacia (si se piensa que se puede cumplir con el régimen) $)^{2}$.

Su nivel de motivación para la protección y la decisión de dejar de fumar, está determinada por medio del equilibrio cuando se comparan sus reconocimientos de amenaza y de cómo sobreponerse.

\section{DISCUSIÓN}

La gran mayoría de los seres humanos se mueve entre las motivaciones de una completa autodeterminación (intrínsecas) hasta la completa 
determinación ambiental (extrínsecas), sin ser estas motivaciones excluyentes. Como entes racionales deciden cómo responder a las fuentes de motivación externas (ambientales) o internas (propias del sujeto), que constituyen el proceso mediador que orienta sus respuestas (García, Domenech 1997)'.

Pero lo que realmente va a determinar un cambio de conducta en el hábito de los fumadores no solo se basa en la autodeterminación o en la determinación ambiental, que conlleva el reconocimiento de la amenaza, sino que es un proceso que también abarca otros ámbitos de la cognición del hombre, y que es fundamental para la motivación de dejar de fumar, nos referimos a que el individuo debe ser capaz de reconocer cuál es la manera de sobreponerse y la autoeficacia, de cualquier tratamiento, lo cual no apunta ninguna de las campañas antitabáquicas de Chile y por ende son un punto muy importante a considerar al momento de crear las estrategias de prevención y acción. Por lo tanto teniendo en cuenta el proceso cognitivo que conlleva un cambio de hábito, cabe destacar que los profesionales de la salud no sólo deben ser capaces de la realización de modelos de prevención sino que también se debe considerar que, deben cumplir con entregar las herramientas necesarias a los fumadores, para que logren controlar este hábito, es decir informar, educar, acompañar y entregar una gama de soluciones que logren satisfacer las necesidades de las personas.

Si analizamos los resultados de las campañas antitabáquicas de Chile, esto nos arroja una luz acerca de lo que ya se ha intentado con los jóvenes y lo que todavía falta por hacer y que es labor de los profesionales, llegar a mejorar la salud por medio de la prevención y el cambio de conducta de nuestros pacientes. Podemos ver que en el caso de las motivaciones extrínsecas que apuntaron a la entrega de premios a los jóvenes que lograban dejar de fumar durante un mes, éstas arrojaron resultados beneficiosos, ya que los ganadores (del concurso organizado por el Ministerio de Salud), fueron capaces de suprimir este hábito tabáquico, lo cual demuestra que el cambio de conductas por medio de motivaciones extrínsecas es asertivo para los jóvenes en Chile y satisface en parte el objetivo de prevención de enfermedades, asociadas al consumo de tabaco.
Ahora si revisamos los resultados del octavo estudio nacional de drogas en población escolar de Chile durante el año 2009, ha dejado en evidencia que históricamente el consumo de tabaco entre los jóvenes ha ido decayendo ${ }^{13}$.

La prevalencia diaria de cigarrillos (20 o más días en el último mes) desciende desde $18,6 \%$ en el año 2003 hasta un rango de entre $15,7 \%$ (2007) y $14,6 \%$ (2009), una caída de 4 puntos porcentuales entre el año 2003 al 2009 equivalentes a $21 \%$ de disminución (Tabla 1).

Tabla 1. Evolución de prevalencia diaria de tabaco en población escolar ${ }^{13}$

\begin{tabular}{|lccc|}
\hline Año & Total & Hombre & Mujer \\
\hline 2003 & 18,6 & 18,3 & 18,9 \\
2005 & 15,3 & 14,2 & 16,5 \\
2007 & 15,7 & 14,7 & 16,7 \\
2009 & 14,6 & 13,6 & 15,7 \\
\hline
\end{tabular}

Lamentablemente estas cifras sólo nos entregan información de disminución de consumo, pero el ¿por qué?, puede estar dado por distintas variables o hipótesis, como que estas campañas realizadas han tenido efecto, que la gente posee una mayor información o que están más concientizados, lo cual demuestra el gran déficit que posee Chile frente a la prevención del consumo de tabaco, mirando estas cifras se puede decir que existen avances, que las políticas y campañas que se han realizado han tenido algunos frutos, pero la tarea es bastante grande y que el rol del profesional es fundamental para el éxito en el avance de disminución del consumo de tabaco.

\section{CONCLUSIÓN}

Como el consumo de tabaco representa la principal causa única prevenible de enfermedad, discapacidad y muerte en el mundo actual ${ }^{12}$. Por lo cual es de vital importancia la eliminación de las conductas del hábito de fumar a edades tempranas. Este cambio de conductas debe considerar que el ser humano actúa en base a diversas motivaciones, que tienen ser satisfechas a la hora de pensar en un 
plan de acción en contra del tabaquismo, considerando como factor esencial la erradicación durante la adolescencia, por ser la edad de inicio y consolidación de la conducta. Y en este ámbito los profesionales de la salud juegan un rol fundamental, ya que ellos deben ser capaces dentro de su quehacer, fomentar e incorporar la prevención.

Otras investigaciones como la realizada en México sobre el impacto de los medios de comunicación, en el proceso de dejar de fumar, mostró que tiene un efecto poderoso frente a la eliminación del hábito tabáquico ${ }^{1}$.

Este estudio sirvió para conocer las motivaciones presentes en la persona a la hora de realizar un cambio de conducta, y cuáles de éstas ya están siendo trabajadas en las campañas antitabáquicas y cuáles quedan pendientes para realizar en la labor profesional.

Una de las limitaciones del estudio fue la escasa información acerca de planes de acción específicos que están siendo realizados por profesionales del área, en prevención del consumo de tabaco.

Dentro de las proyecciones de este estudio sería interesante comprobar que tan eficaz resultaría realizar un plan de acción en donde se abarcara la mayor cantidad de ámbitos de las teorías de las motivaciones, para llegar a un cambio de habito en los fumadores.

\section{REFERENCIAS}

1. Raúl H Sansores, Fernanda Girardo, Fabiola Valdemar, Alejandra Ramírez, Ricardo Sandoval. Impacto de los medios de comunicación masiva en una campaña antitabaco. Salud Pública de México/ vol. 44, suplemento 1. 2002.

2. R.G. Suárez López de Vergara, C. Galván Fernández C. Olivia Fernández, E. Doménech Martínez YF. Barroso Guerrero. Tabaquismo en adolescentes, valores de cotinina en saliva y enfermedad respiratoria. An Esp Pediatr 54: 114119. 2001.

3. Ministerio del Interior Gobierno de Chile 2010. Consejo nacional para el control de estupefacientes (CONACE). Octavo Estudio Nacional de Drogas en Población Escolar de Chile 2009 (Octavo básico a cuarto medio). Disponible en: www.interior.gob.cl.

4. Ivanovic, Daniza M. Factores que inciden en el hábito de fumar de escolares de educación básica y media. Rev. Salud Pública, 31 (1): 30-43. 2000.
5. Encuesta Nacional de la Salud 2011. Gobierno nacional de Chile. Disponible en: www.minsal.gob.cl/port.

6. Ministerio de Salud de Chile. Prevención del consumo de tabaco. Año 2012. Disponible en: www.minsal.cl (Consultado el 20 de abril de 2012).

7. Verónica Behn Theune, Mónica Cruz Pedreros, Julia Huaiquián Silva, Rina Naveas González, Hernán Sotomayor León. Motivations of nonsmokers for continuing not to smoke. Ciencia y Enfermeria ix (1): 131-37, 2003.

8. Hugo Amigo, Marcia Erazo, Manuel Oyarzún G, Sergio Bello, Armando Peruga. Smoking and chronic obstructive pulmonary disease: atributable risk determination. Rev Méd Chile 2006; 134: 1275-1282.

9. Berta Lidia Nuño-Gutiérrez, Jose ÁlvarezNemegyei, Eduardo A. Madrigal-De León. Effect of an anti-tobacco intervention in high school students from Guadalajara, Mexico. Salud Ment v.31 n.3 México mayo/junio. 2008.

10. F. Carrion Valero, C. Pellicer Ciscar. El tabaquismo pasivo en la infancia; nuevas evidencias. Prevención del tabaquismo vol. $4 \mathrm{n}^{\circ} 1$, eneromarzo 2002.

11. Departamento de Epidemiología Ministerio de Salud de Chile 2005. Encuesta Mundial sobre Tabaquismo en Jóvenes. Chile, 2003. Disponible en: www.epi.minsal.cl

12. Marta Pernas Gómez y Lourdes Arencibia Flores. Efectos sobre la salud de la exposición crónica al humo del tabaco en fumadores y no fumadores. Rev Cubana med gen Integr 2000; 14(2): 180-4.

13. Verónica Behn T, Hernán Sotomayor, Mónica Cruz P, Rina Naveas. Motivations to quit Smoking among former smoker university students and workers. Rev. Méd. Chile v.129 n.5 Santiago. Mayo 2001.

14. Hogg, Vaughan. Psicología social 2008.

15. Arsheli Rojas Garrido, Gerardo López Pérez, Socorro Orozco Martínez, Enrique Guerrero Sánchez, José G Huerta López. Tabaquismo y desarrollo de alergia durante la infancia. Mecanismos fisiopatológicos. Alergias, asmas e inmunología pediátrica, Vol. 10, Núm. 2, MarzoAbril 2001. 